This is a self-archived version of an original article. This version may differ from the original in pagination and typographic details.

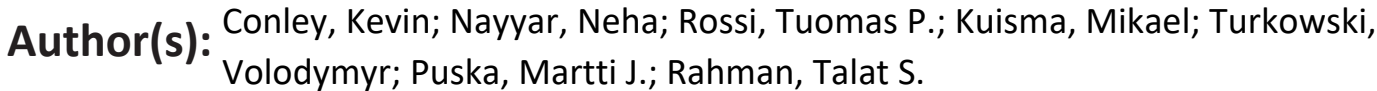

Title: Plasmon excitations in chemically heterogeneous nanoarrays

Year: 2020

Version: Published version

Copyright: @ $\odot 2020$ American Physical Society

Rights: In Copyright

Rights url: http://rightsstatements.org/page/InC/1.0/?language=en

Please cite the original version:

Conley, K., Nayyar, N., Rossi, T. P., Kuisma, M., Turkowski, V., Puska, M. J., \& Rahman, T. S. (2020). Plasmon excitations in chemically heterogeneous nanoarrays. Physical Review B, 101(23), Article 235132. https://doi.org/10.1103/PhysRevB.101.235132 


\title{
Plasmon excitations in chemically heterogeneous nanoarrays
}

\author{
Kevin Conley $\odot,{ }^{1}$ Neha Nayyar, ${ }^{2}$ Tuomas P. Rossi, ${ }^{3}$ Mikael Kuisma, ${ }^{4}$ Volodymyr Turkowski, ${ }^{2}$ \\ Martti J. Puska ${ }^{0},{ }^{5}$ and Talat S. Rahman $\mathbb{1 0}^{2,5}{ }^{*}$ \\ ${ }^{1}$ Department of Applied Physics, QTF Centre of Excellence, Aalto University School of Science, P.O. Box 11100, FI-00076 Aalto, Finland \\ ${ }^{2}$ Department of Physics, University of Central Florida, Orlando, Florida 32816, USA \\ ${ }^{3}$ Department of Physics, Chalmers University of Technology, SE-412 96 Gothenburg, Sweden \\ ${ }^{4}$ Department of Chemistry, Nanoscience Center, University of Jyväskylä, FI-40014 Jyväskylä, Finland \\ ${ }^{5}$ Department of Applied Physics, Aalto University School of Science, P.O. Box 11100, FI-00076 Aalto, Finland
}

(Received 18 November 2019; revised manuscript received 14 March 2020; accepted 20 May 2020; published 11 June 2020)

\begin{abstract}
The capability of collective excitations, such as localized surface plasmon resonances, to produce a versatile spectrum of optical phenomena is governed by the interactions within the collective and single-particle responses in the finite system. In many practical instances, plasmonic metallic nanoparticles and arrays are either topologically or chemically heterogeneous, which affects both the constituent transitions and their interactions. Here, the formation of collective excitations in weakly $\mathrm{Cu}$ - and Pd-doped $\mathrm{Au}$ nanoarrays is described using time-dependent density functional theory. The additional impurity-induced modes in the optical response can be thought to result from intricate interactions between separated excitations or transitions. We investigate the heterogeneity at the impurity level, the symmetry aspects related to the impurity position, and the influence of the impurity position on the confinement phenomena. The chemically rich and symmetry-dependent quantum mechanical effects are analyzed with transition contribution maps demonstrating the possibility to develop nanostructures with more controlled collective properties.
\end{abstract}

DOI: 10.1103/PhysRevB.101.235132

\section{INTRODUCTION}

The interaction of light with finite metallic nanoparticles and arrays can excite localized surface plasmon resonances (LSPR). The collective oscillation of electrons emerges from early descriptions of a degenerate electron gas [1]. Recently, time-dependent density functional theory (TD-DFT) based methods are applied to increasingly large systems, such as $\mathrm{Au}$ [2] and Ag nanoparticles [3-5]. The prospect of improving the understanding of the interaction among the electron-hole pairs within plasmons has motivated exploration for photocatalysis [6] or hot electron generation at metal-semiconductor interfaces $[7,8]$. Goal-oriented studies necessitate investigation of nanosystems which are not topologically or chemically homogeneous for their unique pathways of plasmon formation and decay. Introducing heterogeneity will affect the extended electronic structure and its excitations.

Collective electron excitations, such as LSPR in nanoparticles and molecular plasmons in smaller atomic arrangements or molecules, can be analyzed as interactions between contributing electron-hole transitions. Indeed, the identification and analysis of the electron-hole contributions in excitation peaks of photoabsorption spectra has become easily attainable with analysis methods $[9,10]$ alongside TD-DFT. A transition contribution map (TCM) visually parses the energy of the individual electron-hole transition contributions and informs about the interactions between electron-hole pairs [10,11].

\footnotetext{
*talat.rahman@ucf.edu
}

Plasmons and single-particle excitations are also differentiated by the distinct dependence on the Coulomb interaction scaling parameter $\lambda$ [9]. Moreover, the generalized plasmonicity index is an analogous method to quantify the contributions from low-energy transitions [12] which shows clearly the formation of the LSPR [13].

TCMs are increasingly practical for the analysis of plasmonic systems $[10,11,14-17]$. TCMs of the intense photoabsorption excitations have provided fundamental insight into how the individual electron-hole contributions are pieced together from the nodal structure of the delocalized $s p$ states within homogeneous metallic arrays of varying width [16]. States containing more transverse nodes become more energetically stable and occupied in wider arrays. The collectivity of the so-called molecular plasmon grows as more transitions are possible.

Molecular plasmons exhibit effects from finite-size quantum confinement, symmetry, and avoided crossings within nanosystems such as metallic clusters [18-24], arrays $[16,25,26]$, chains [27-29], and conjugated and aromatic hydrocarbons [30-34]. As evidenced by previous computational studies $[16,25,26]$, double-chain atomic arrays of metals, which we consider in this study, contain many of the physical and chemical phenomena which are central to any molecular plasmon. They exhibit plasmonic excitations arising from a clearly identifiable nodal structure and a more discernible collective character than a single chain. Further, two-dimensional arrays require fewer atoms to study long structures without needlessly increasing computational tractability. As shown below, these advantages become clear when chemical hetero- 
geneity changes the symmetry and electronic structure and resulting formation of molecular plasmons in arrays containing substitutional dopants.

In this paper, we calculate the optical response of weakly doped free-standing double-chain arrays using TD-DFT. The geometries and the computational methods employed in the TD-DFT calculations are described in Sec. II. In Sec. III, the effects within homogeneous Au double-chain arrays are briefly discussed. Then, we describe how the electronic structure and plasmonic features change when the double-chain arrays are weakly doped with either $\mathrm{Cu}$ or $\mathrm{Pd}$. The chemical heterogeneity introduces phenomena that are distinct from those found earlier in arrays containing mixed homonuclear chains or homogeneous arrays of varying size. The origins of the new phenomena are clarified with some of the analysis methods described above.

\section{METHODS}

Gold $(\mathrm{Au})$ atomic wires are constructed 10 to 20 atoms in length. One atom in the chain is doped by substituting the $\mathrm{Au}$ atom with either a noble $(\mathrm{Cu})$ or transition metal $(\mathrm{Pd})$ at different positions along the chain, and then two similarly doped chains are assembled to form arrays of heteronuclear chains up to $5.5 \mathrm{~nm}$ in length (cf., Fig. 1). For brevity, the finite-size double-chain array atomic nanosystems are called arrays. A single Au chain 19 atoms in length and doped with either one $\mathrm{Cu}$ or one $\mathrm{Pd}$ atom was also considered. To mimic supported nanostructures in realistic systems, a square lattice is assumed and the bond length is set to $2.89 \AA$, as measured in experimentally realized Au chains on the $\mathrm{NiAl}(110)$ substrate [35]. Note that in a related earlier work it was found that the absorption spectra are not very sensitive to changes in the $\mathrm{Au}$ bond length in the range 2.5 to $2.89 \AA$ [36]. The free-standing model allows a straightforward identification and analysis of the nodal structure and discrete electron-hole transitions. The emphasis on simple geometric and chemical modification results in clear changes in the photoabsorption spectra and electronic structure which can guide analyses of measured systems.

The electronic structure of the free-standing doped nanostructures was calculated with DFT [37,38] using the solidstate modified GLLB-SC exchange-correlation potential [39] which accurately describes the energy level positions of the $d$ states in noble metals $[40,41]$. We employ the GPAW code package [3,42-44] based on the projector-augmented-wave (PAW) method [45] and utilize the ASE package [46]. Electronic wave functions are expanded as linear combinations of atomic orbitals (LCAO) [47] and occupied with less than $0.05 \mathrm{eV}$ Fermi-Dirac smearing. Spin-orbit coupling was not considered, but relativistic effects are included at the scalarrelativistic level in the PAW setups. The pseudo wave functions are evaluated in real space with a grid spacing of 0.3 $\AA$ and the electron density and potentials are evaluated on a doubly fine $0.15 \AA$ grid. The arrays are surrounded by at least $6 \AA$ of vacuum and the Hartree potential is evaluated on a larger and coarser grid with at least $48 \AA$ of vacuum.

The optical response of each array and single chain was calculated with TD-DFT [48]. The $10 \times 2$ arrays and single chains were calculated within the Casida formulation of
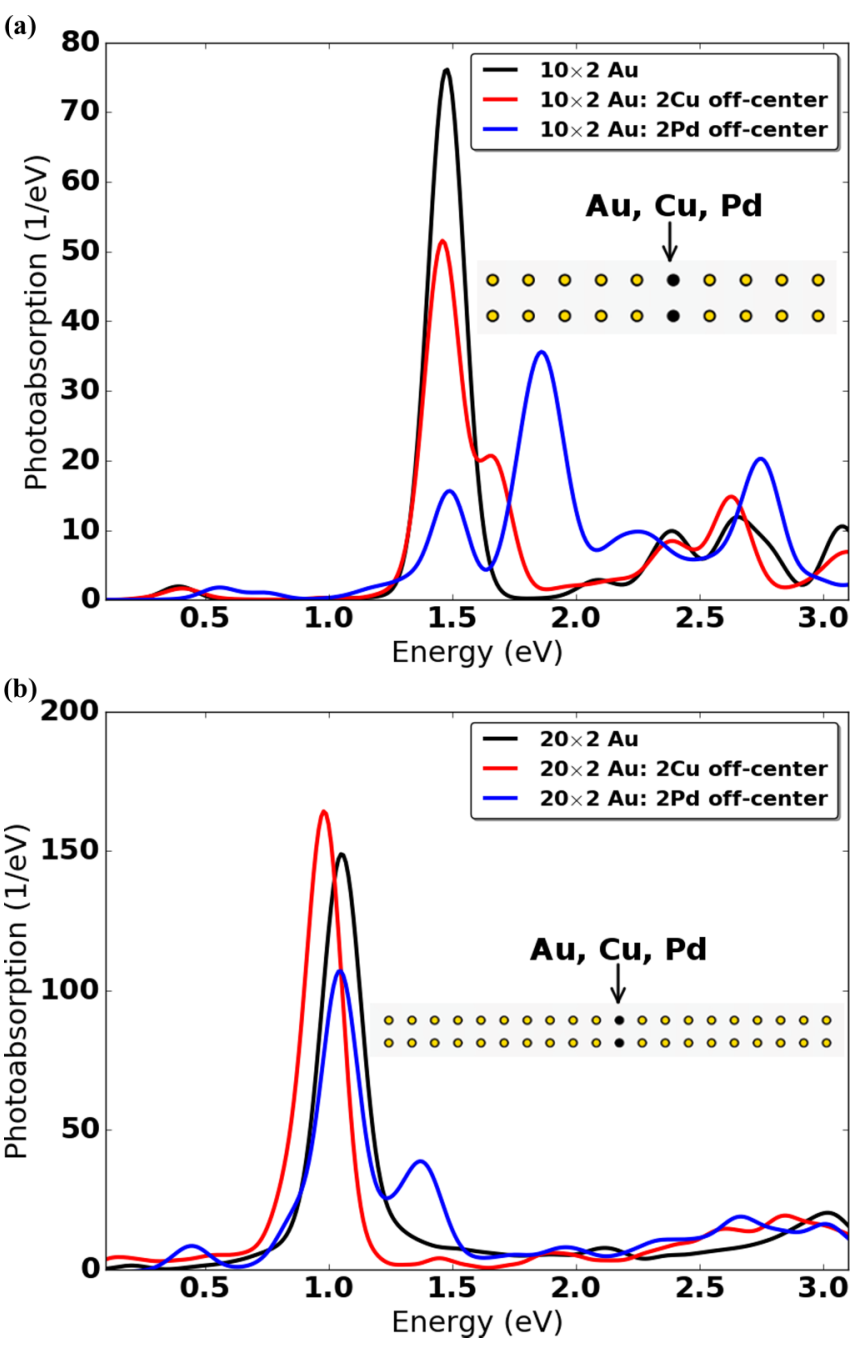

FIG. 1. Photoabsorption spectra of weakly doped double-chain arrays. Photoabsorption spectra of a $10 \times 2$ (a) and $20 \times 2$ (b) double-chain $\mathrm{Au}$ array doped with $\mathrm{Pd}$ or $\mathrm{Cu}$ within the array (off center) compared to that of a corresponding pristine $\mathrm{Au}$ array. There is one impurity atom in each chain, and the impurities are nearest neighbors.

linear-response TD-DFT [49] and the LCAO time propagation (TP) TD-DFT code [3]. In the Casida approach, the matrix eigenvalue equation [49]

$$
\Omega \mathbf{F}_{I}=\omega_{I}^{2} \mathbf{F}_{I}
$$

is solved to yield the excitation energies $\omega_{I}$ and corresponding Casida eigenvectors $\mathbf{F}_{I}$. The matrix $\Omega$ is constructed in electron-hole space such that the Kohn-Sham (KS) excitations $i \rightarrow a$ and $j \rightarrow b$ are written as

$$
\Omega_{i a, j b}=\omega_{i a}^{2} \delta_{i a, j b}+2 \sqrt{f_{i a} \omega_{i a}} \lambda K_{i a, j b} \sqrt{f_{j b} \omega_{j b}},
$$

where $f_{i a}$ is the occupation-number difference, $\omega_{i a}=\epsilon_{a}-\epsilon_{i}$ is the KS eigenvalue difference, and $K_{i a, j b}$ represents the firstorder interaction ("coupling") between the excitations $i \rightarrow a$ and $j \rightarrow b$. The dimensionless scaling factor $\lambda$ provides a transformation from the noninteracting framework at $\lambda=0$ to the fully interacting framework at $\lambda=1$ [9]. Note that the transformation scales the Hartree-exchange-correlation 
kernel with $\lambda$ and constitutes a possible path for intermediate values. The path depicts the renormalization of the excitations with other transitions and allows analysis of the nature of the excitations [9]. Here, the adiabatic PBE [50] kernel is used. The Gaussian broadening of the spectra is $\sigma$ $=0.07 \mathrm{eV}$.

In the TP TD-DFT approach [3], the electron wave functions are evolved after an initial external electric (in dipole approximation) $\delta$ pulse [51] along the long wire axis. The time-dependent induced density provides the time-dependent dipole moment from which the dynamical polarizability and the ensuing frequency-dependent photoabsorption spectrum are determined. The photoabsorption spectrum with Gaussian broadening of $\sigma=0.07 \mathrm{eV}$ is obtained using a total propagation time of $30 \mathrm{fs}$ and a time step of 10 as. The noninteracting spectra were calculated with the interaction strength parameter $(\lambda)$ equal to 0 . The spectrum calculated using the TP method with full interaction was equivalent to the optical spectrum from the Casida formulation.

Both for the Casida and TP formulations of TD-DFT, the linear response of the real part of the KS density matrix $\delta \rho_{i a}(\omega)$ and the corresponding dipole matrix elements allow the photoabsorption spectrum to be decomposed into contributions from individual discrete electron-hole transitions $[10,11]$. The different discrete electron-hole transitions are denoted by spots on a two-dimensional map [cf., Fig. 2(a)] with an intensity proportional to the normalized photoabsorption decomposition weight of the KS electron-hole transition $\left(S_{i a} / S\right)$ [see Eqs. (17) and (21) in Ref. [10]]. The red (blue) color of the spots corresponds to the positive (negative) sign of the photoabsorption decomposition of the transition. The extent is given by a two-dimensional Gaussian broadening function $\left(\sigma_{\mathrm{TCM}}=0.04 \mathrm{eV}\right)$ and is distinct from the broadening of the photoabsorption spectrum $\sigma$. In this paper, the initially unoccupied and initially occupied energies $\epsilon_{\mathrm{u}}$ and $\epsilon_{\mathrm{o}}$ and density of states (DOS) are given on the vertical and horizontal axes of TCM, respectively. The photoabsorption energy is noted as $\omega$ and spots on the line $\omega=\epsilon_{\mathrm{u}}-$ $\epsilon_{\mathrm{o}}$ correspond to the electron-hole transition contributions which are not affected by the Hartree-exchange-correlation interactions.

The induced density can be decomposed into partial densities corresponding to the different electron-hole transition contributions. The density contribution from the transition from an occupied ground state $\psi_{i}^{(0)}(\mathbf{r})$ to an unoccupied ground state $\psi_{a}^{(0)}(\mathbf{r})$ is given by

$$
\delta n_{i a}(\mathbf{r}, \omega)=2 \psi_{i}^{(0)}(\mathbf{r}) \psi_{a}^{(0) *}(\mathbf{r}) \delta \rho_{i a}(\omega)
$$

and the density contributions sum to the total induced electron density $\delta n(\mathbf{r}, \omega)=\sum_{i a}^{\text {eh }} \delta n_{i a}(\mathbf{r}, \omega)$. Further theoretical details about these analysis tools can be found elsewhere [10].

Some of the nanowire systems were also calculated using Gaussian 03 [53] with a B3PW91 hybrid functional [54-56] and a LanL2DZ basis set [57]. The main features of the photoabsorption spectra were consistent with the results herein (see Supplemental Material [58]).
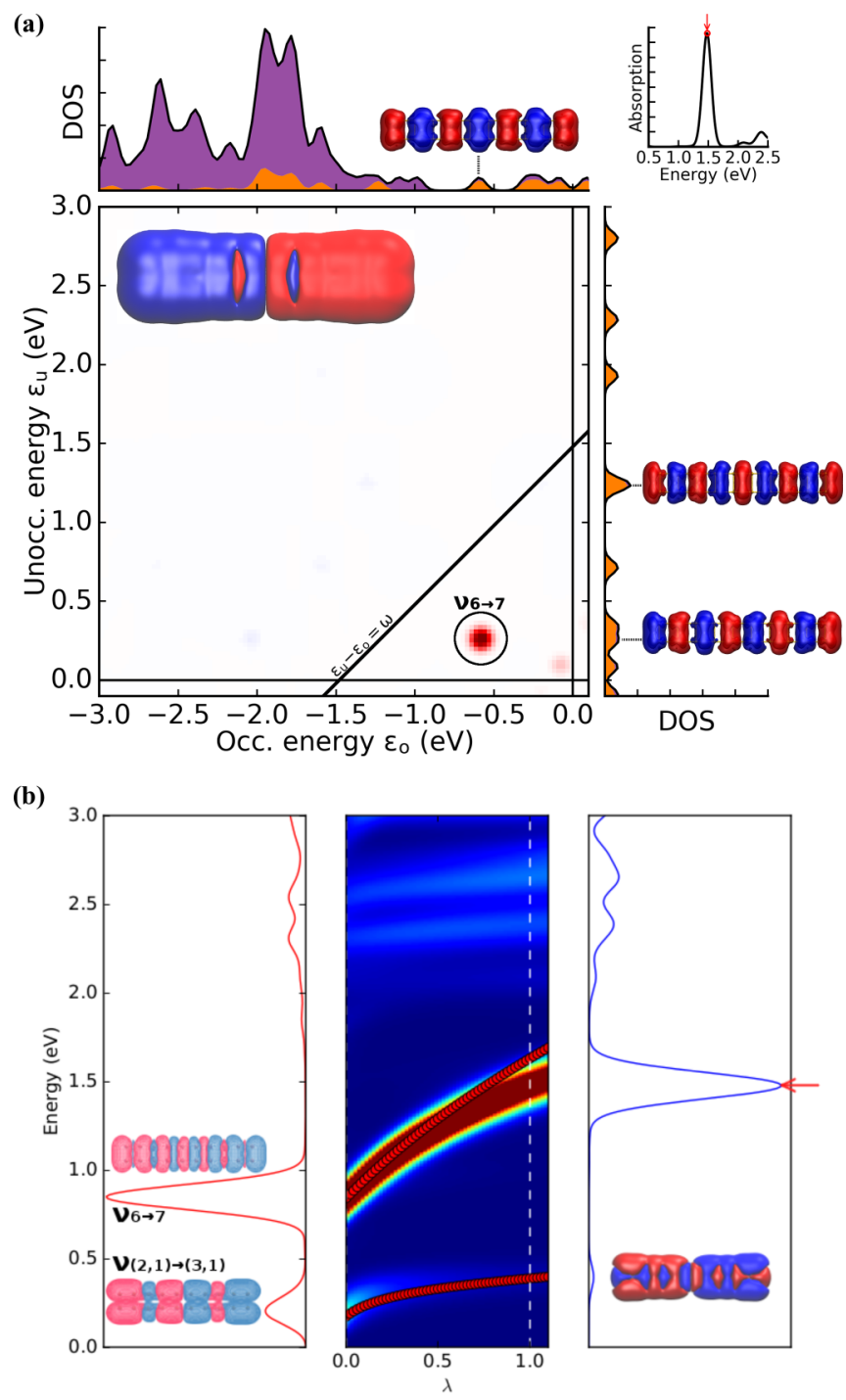

FIG. 2. Homogeneous $10 \times 2 \mathrm{Au}$ array. (a) Contributions to the plasmonic excitation at $\omega=1.48 \mathrm{eV}$. The total induced density is shown in the inset and relevant $\mathrm{KS}$ orbitals are provided adjacent to the density of states. (b) $\lambda$-dependent photoabsorption spectra of the array. The red dots denote the $\lambda$-dependent pseudoexcitation energies of two interacting KS transitions ( $v_{6 \rightarrow 7}$ and the HOMO $\rightarrow$ LUMO). The partial induced density of each KS transition is shown adjacent to the noninteracting spectrum, and the total induced density of the low-energy excitation is shown adjacent to the spectrum with full interaction. The arrow indicates the photoabsorption mode analyzed in the TCM.

\section{RESULTS AND DISCUSSION}

Nanoalloy arrays contain numerous physical and chemical aspects fundamental to molecular plasmonics in chemically heterogeneous systems. In this section, the photoabsorption modes in the spectra of $10 \times 2$ and $20 \times 2 \mathrm{Au}$ arrays weakly doped with $\mathrm{Cu}$ or Pd (Fig. 1) are described qualitatively. In the subsequent subsections, TCMs and the $\lambda$ scaling of 
the collective response are used to examine the interactions among the electron-hole contributions and their influence on the plasmon formation.

Molecular arrays with varying number of chains have been examined elsewhere [16,25,26] and have a nonmonotonic shift of the plasmon frequency due to the evolving subband structure and nodal planes. TCMs demonstrated an increase in collectivity as the transitions within each contributing subband become more numerous [16]. Double-chain Au arrays were shown to have a strong photoabsorption mode from predominantly the subband with zero transverse nodes (with nodal surfaces parallel to the chain axis) [16]. Strong wellresolved peaks are seen in Fig. 1 for the absorption spectra of the $10 \times 2$ and $20 \times 2$ arrays which are the basic model systems considered in this paper.

Elongating the arrays will modify the subband structure and shift the plasmon frequency. The $10 \times 2 \mathrm{Au}$ array has an intense photoabsorption mode at $1.48 \mathrm{eV}$ as seen in Figs. 1(a) (black line) and 2. The photoabsorption mode redshifts to $1.05 \mathrm{eV}$ in the elongated $20 \times 2$ array as seen in Figs. 1(b) (black line) and 3(a).

Introducing chemical heterogeneity to the Au array by doping can shift the energy of the mode or generate additional strong photoabsorption modes. For example, a split of the plasmon mode is observed when $\mathrm{Cu}$ doping is used within the small array [Fig. 1(a)]. The arrays are an even number of atoms in length and the dopants are situated just off center the middle of the array as shown in the cartoon. The modes coalesce into an unresolved strong mode by lengthening the $\mathrm{Cu}$-doped array [Fig. 1(b)]. The situation is different for Pddoped arrays; two or more additional peaks form irrespective of the array length. This behavior differs qualitatively also from the case in which a whole chain of Pd atoms replaces a chain of $\mathrm{Au}$ atoms in a mixed double-chain array, i.e., the Pd row substitution quenches the plasmon of the Au double chain [16].

The photoabsorption spectra of homogeneous and doped single chains 19 atoms in length are shown in Fig. S1 [58]. The second chain focuses the electron density between the chains, which leads to a blueshift of the plasmon energy from homogeneous single- to double-chain arrays. The shift in plasmon energy for arrays of vary width is reflected in the work function and electron density spillout and is discussed in detail elsewhere [16]. A high-energy shoulder appears in the photoabsorption spectrum of a $\mathrm{Cu}$-doped single chain when the dopant is positioned in the interior (Fig. S1A [58]). A single intense mode appears when a $\mathrm{Pd}$ atom is positioned at the end of a single Au chain (Fig. S1B [58]). The plasmon energy shifts to a higher energy when the Pd is located in the middle of the chain. Details regarding the influence of a single $\mathrm{Cu}$ or Pd dopant on the photoabsorption spectra of a single chain 19 atoms in length are provided in the Supplemental Material, Figs. S2 and S3 [58].

In the following sections, we discuss how the plasmonic features of $\mathrm{Cu}$ - or Pd-doped Au arrays are determined by their electronic structures and point-group symmetries. The hybridization of states in nanoscale particles and interactions within the homogeneous and chemically heterogeneous arrays are identified and characterized using TCMs, group theory, and the $\lambda$-scaling approach.
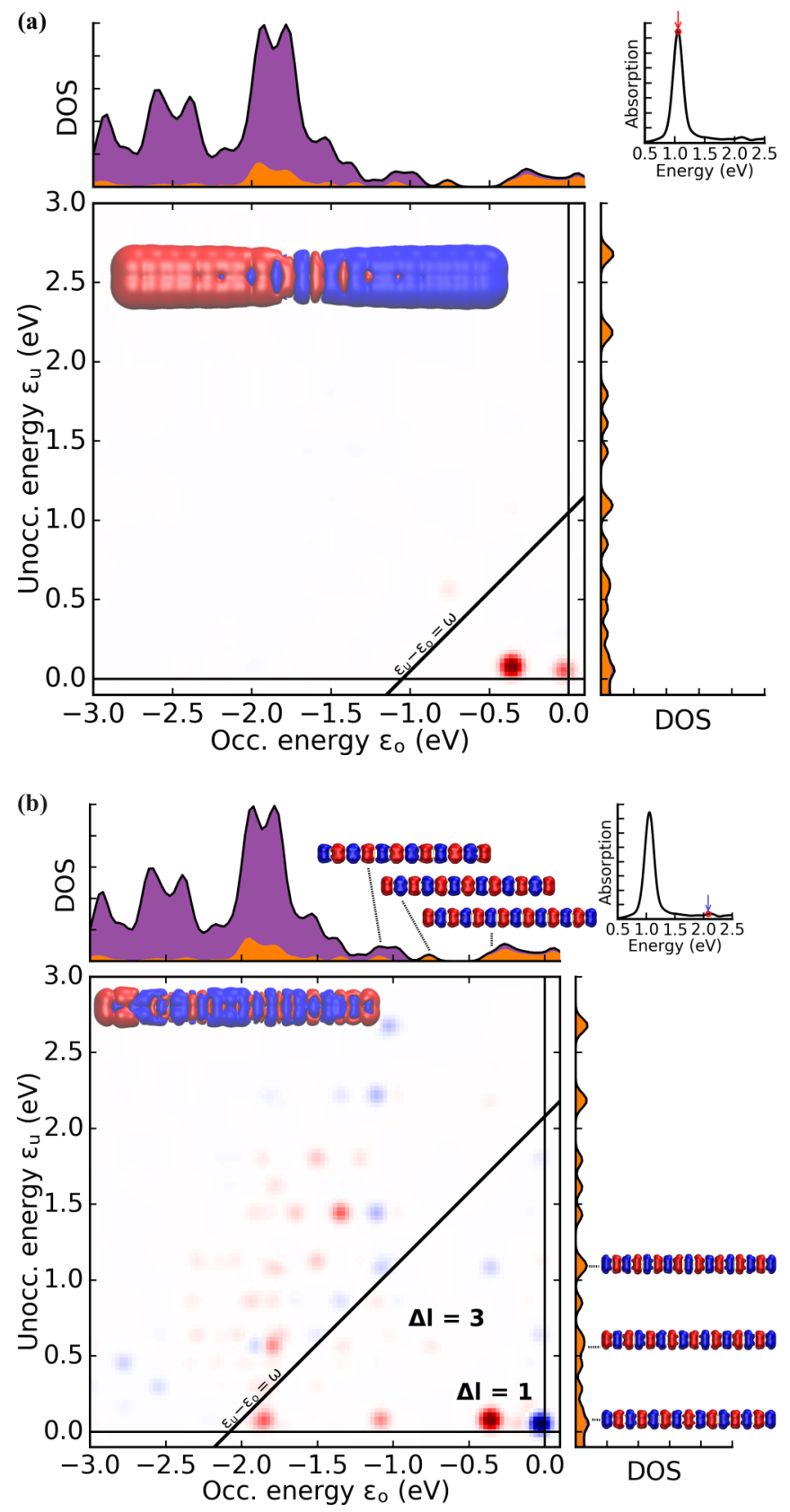

FIG. 3. Homogeneous $20 \times 2 \mathrm{Au}$ array. TCM of the photoabsorption excitations at (a) 1.05 and (b) $2.08 \mathrm{eV}$. The total induced density of each excitation is shown in the inset and relevant $\mathrm{KS}$ orbitals are provided adjacent to the density of states.

\section{A. Homogeneous double-chain array}

In this section, the plasmon formation within homogeneous Au arrays is described using TD-DFT methods and TCMs of the KS transition contributions. The symmetry and interaction between the transition contributions in the chemically pure arrays form the basis of the later discussion of chemically heterogeneous systems.

The undoped $10 \times 2$ Au double-chain array has a single intense mode at $\omega=1.48 \mathrm{eV}$ and the construction of the electronic excitation from the individual KS transition contributions is illustrated in the TCM in Fig. 2(a). The electronic 
excitation consists of a contribution from the KS transition between $s p$-delocalized states with six and seven longitudinal nodes (with nodal surfaces transverse to the chain axis), $v_{6 \rightarrow 7}$ or $(6,0) \rightarrow(7,0)$ wherein $(l, t)$ denotes the number of longitudinal $l$ and transverse $t$ nodes. This contribution is shown as a bright red dot on the TCM. The transition is clearly below the line $\epsilon_{\mathrm{u}}-\epsilon_{\mathrm{o}}=\omega$ which reflects the increase of the excitation energy due to the interaction $[9,10]$. The KS orbitals of the initial and final states are shown adjacent to the TCM. In double-chain arrays, the transitions within other subbands are weak due to the presence of a node between the two chains. Nevertheless, the HOMO $\rightarrow$ LUMO transition, i.e., $(2,1) \rightarrow(3,1)$, has a minor contribution and is dimly visible in the lower right corner. The longitudinal nodal difference of both contributing transitions is unity producing a collective excitation with the dipolar total induced density shown in the TCM inset.

The evolution of the photoabsorption spectrum with the interaction strength parameter $\lambda$ is shown in Fig. 2(b). See Eq. (2) in the Methods section for details. A dark red stripe originating at $v_{6 \rightarrow 7}$ blueshifts as $\lambda$ increases. This shift has been used to indicate plasmonicity in similar atomic arrays and molecules $[9,13,30]$. The weak mode, initially at $0.17 \mathrm{eV}$, gradually diminishes while blueshifting with increasing $\lambda$. The light horizontal stripes around $2.5 \mathrm{eV}$ are formed by the interband transitions from the $d$ band. These transitions are marginally affected by the interaction parameter indicating single-particle character.

The interaction between specific KS transitions can be examined by renormalizing the excitation energies of a subset of KS transitions. The renormalized excitation energies consisting of a subset of KS transitions are called pseudoexcitations. The $\lambda$ evolution of the pseudoexcitation energies consisting of only two transition contributions, $v_{6 \rightarrow 7}$ and the HOMO $\rightarrow$ LUMO, are drawn as red dots and superimposed onto the full set of possible interacting transitions in Fig. 2(b). The two KS transitions interact to form symmetric and antisymmetric combinations at higher and lower energies, respectively. The two pseudoexcitation energies blueshift to about 0.5 and $1.5 \mathrm{eV}$ at $\lambda=1$. The spectra with full interaction also include the interaction with the background transitions. The background transitions have low oscillator strengths and weak interaction, and yet lower the energy of the dark red stripe compared to the energy of the red dots of the symmetric pseudoexcitation.

The interference between the two first-order transition contributions is destructive in the antisymmetric combination and constructive in the symmetric combination. The constructive addition of dipoles has been used to gauge the coherence of an excitation [24]. While both excitations scale with $\lambda$, the very weak excitation at about $0.4 \mathrm{eV}$ does not have the coherence required to be a plasmonic excitation.

Elongating the double-chain array to $20 \times 2$ redshifts the intense photoabsorption mode to $1.05 \mathrm{eV}$. The transition energy (eigenvalue difference of the KS states in the transition) of the dominant contribution to the molecular plasmon decreases in the longer homogeneous array. In the $10 \times 2$ array, the first-order, fundamental transition, i.e., having $\Delta l=$ 1 and zero transverse nodes, is $(6,0) \rightarrow(7,0)$. Whereas in a $20 \times 2$ array the fundamental transition occurs from the $(13,0)$ to the $(14,0) \mathrm{KS}$ orbital [Fig. 3(a)]. The transition energies are consistent with those of a noninteracting homogeneous electron gas, which has been discussed for molecular plasmons elsewhere [9]. In TCMs the decrease of the transition energies in longer arrays manifests as a shift of the contribution toward the lower right corner.

The decreased energy spacing of the $s p$-delocalized states in the longer arrays can be observed in the DOS alongside the respective TCMs. For example, the virtual $s p$ states are more dense in the longer array than the well-separated states in the shorter array. The KS orbitals $(8,0)$ and $(15,0)$ of the $10 \times 2$ and $20 \times 2$ array have been identified in the TCMs in Figs. 2(a) and 3(b), respectively.

Even-order transitions in the centrosymmetric arrays are parity forbidden. Dipole-forbidden dark modes, such as the second order $v_{6 \rightarrow 8}$ in the homogeneous $10 \times 2$ array, are absent in the noninteracting spectrum [Fig. 2(b), left panel]. The three possible third-order transitions, however, are parity allowed. These higher-order transitions are visible in the TCM when they mix with the interband single-particle excitations at higher energies. For example, the TCM at $2.08 \mathrm{eV}$ is shown in Fig. 3(b).

\section{B. Cu-doped double-chain array}

In this section chemical heterogeneity is introduced to the homogeneous $\mathrm{Au}$ arrays by substituting one $\mathrm{Au}$ in each chain with $\mathrm{Cu}$ (cf., Fig. 1). We first explain the symmetry-dependent influence of the chemical heterogeneity on the electronic structure and how doping introduces higher-order transitions. Then, we analyze the interaction between the multiorder KS transitions and the consequence on the collective excitations.

Doping a $10 \times 2 \mathrm{Au}$ array with $\mathrm{Cu}$ off center produces two photoabsorption modes at 1.46 and $1.66 \mathrm{eV}$ as seen in Fig. 1(a) (red line). The transition contributions to the electronic excitation in the higher-energy excitation in the doped array are illustrated in Fig. 4(a). See Fig. S4A [58] for the TCM of the lower-energy mode of the doped array. Like the homogeneous array discussed above, the $\mathrm{Cu}$-doped array hosts a KS transition between $s p$-delocalized states with six and seven longitudinal nodes, i.e., $v_{6 \rightarrow 7}$. The KS transition $v_{6 \rightarrow 7}$, shown in the TCM, strongly contributes to both excitations of the doped array. In addition, there are contributions from two additional transitions, labeled $\nu_{5 \rightarrow 7}$ and $v_{6 \rightarrow 8}$. These contributions are from second-order transitions between the $s p$-delocalized states $(5,0) \rightarrow(7,0)$ and $(6,0) \rightarrow(8,0)$. The corresponding KS transitions in the pure centrosymmetric array are dipole-forbidden dark transitions. By being positioned off center the $\mathrm{Cu}$ dopants break the symmetry of the pure array and change the point group from $D_{2 \mathrm{~h}}$ to $C_{2 \mathrm{v}}$ and transitions between states with an even-number difference in longitudinal nodes are no longer dipole forbidden.

The effect of the dopants on the initial and final wave functions of $v_{5 \rightarrow 7}$ are depicted in Fig. 4(c), left. $\psi_{(5,0)}$ and $\psi_{(7,0)}$ of the homogeneous and $\mathrm{Cu}$-doped array are plotted $3 \AA$ off the array edge and in the plane of the array. The sinusoidal pattern depicts the five and seven longitudinal nodes of the $s p$-delocalized states. The valence electron configurations of atomic $\mathrm{Cu}$ and $\mathrm{Au}$ are $4 s^{1} 3 d^{10}$ and $6 s^{1} 5 d^{10}$, respectively. Thus, doping the $\mathrm{Au}$ array with $\mathrm{Cu}$ breaks the symmetry of 

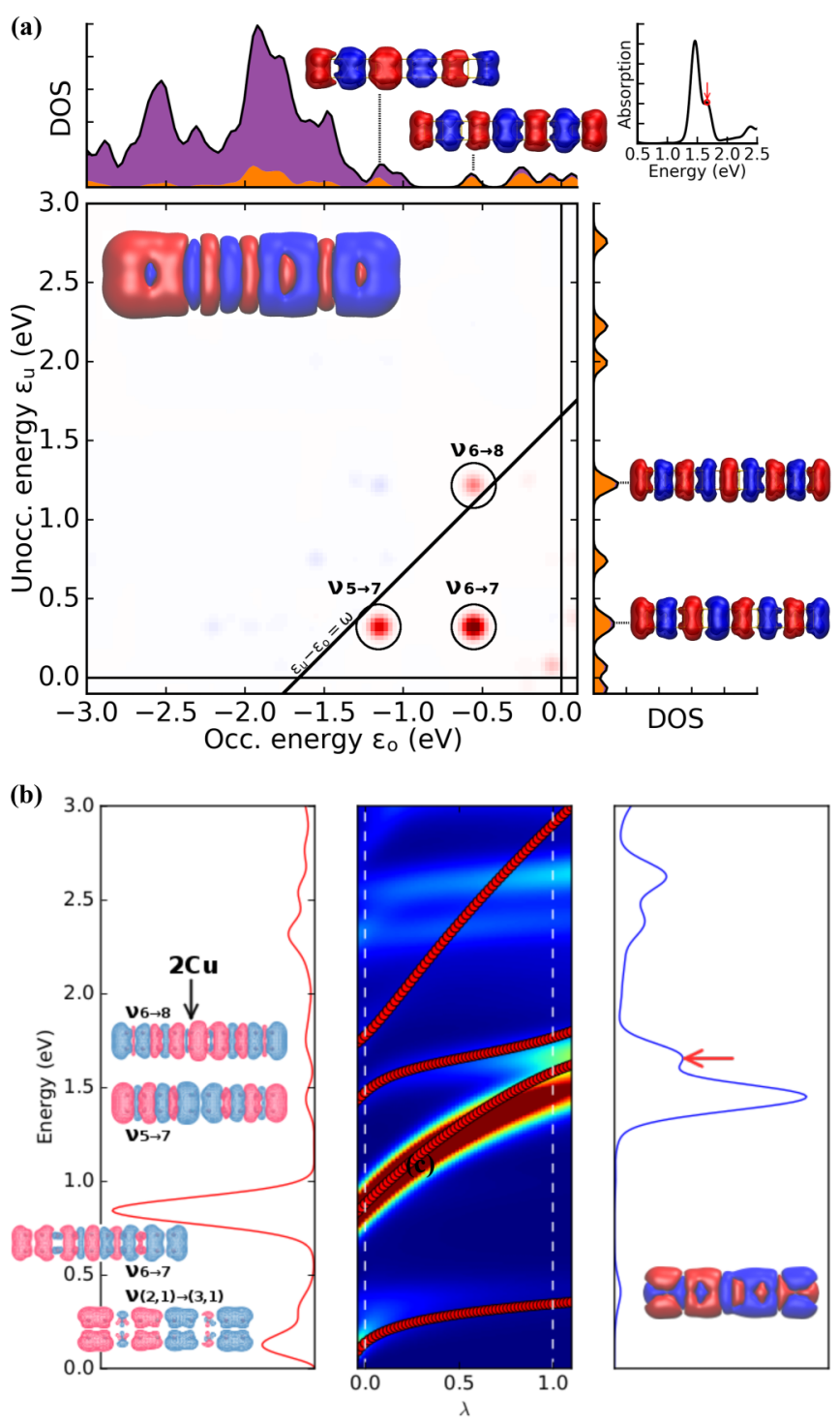

(c) $\psi(7,0)$ :
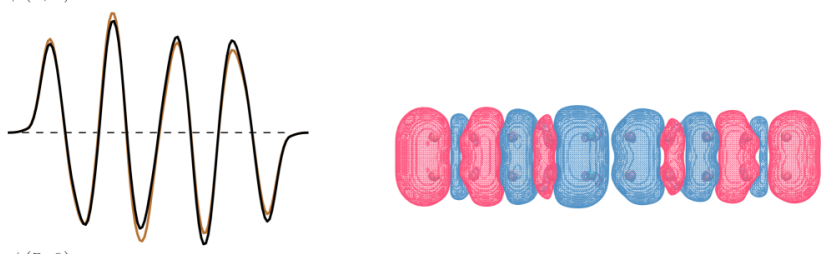

$\psi(5,0)$ :
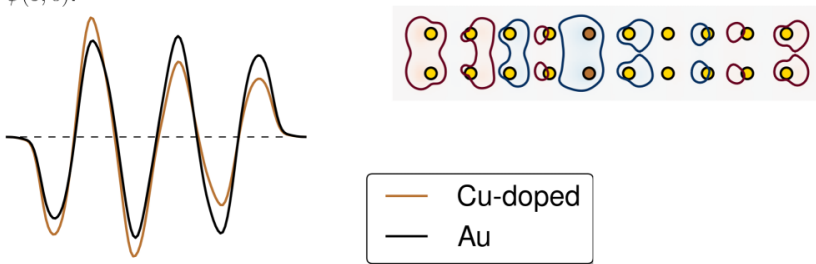

FIG. 4. Off-center $\mathrm{Cu}$-doped $10 \times 2$ array. There is one impurity atom in each chain and the impurities are nearest neighbors as shown in Fig. 1. (a) Contributions to the doping-induced photoabsorption mode at $\omega=1.66 \mathrm{eV}$. The total induced density is shown in the inset and relevant KS orbitals are provided adjacent to the density of states. (b) $\lambda$-dependent photoabsorption spectra of the $\mathrm{Cu}$-doped array. The red arrow indicates the photoabsorption mode analyzed in the TCM. The partial induced density of each KS transition and total induced the magnitude of the wave functions along the array axis, i.e., along the direction of the external electric field, while maintaining the nodes. The influence of doping is also observed in the isosurface plots of the $\mathrm{KS}$ orbitals and in the partial transition density between these states is given in Fig. 4(c), right as a three-dimensional representation (top) and as a slice $0.75 \AA$ above the plane of the array (bottom). There is an overall dipole because the magnitude on the left side of the array is greater than that on the right side of the array.

Two additional weak modes from $v_{5 \rightarrow 7}$ and $v_{6 \rightarrow 8}$ appear in the noninteracting spectrum in the left panel of Fig. 4(b). Each mode occurs at the energy corresponding to the respective eigenvalue difference of the KS states in the transition $(\Delta \epsilon$ $=1.47$ and $1.78 \mathrm{eV}$ ). The magnitudes of $\nu_{5 \rightarrow 7}$ and $\nu_{6 \rightarrow 8}$ are weak relative to that of $\nu_{6 \rightarrow 7}$, which is the strong mode at $\Delta \epsilon=0.88 \mathrm{eV}$. The large overall dipole moment of $v_{6 \rightarrow 7}$ is evident in the well-separated partial induced density shown adjacent to the noninteracting spectrum. $v_{5 \rightarrow 7}$ and $v_{6 \rightarrow 8}$ have weak dipole moments due to the even order of the transitions.

Once the even-order KS transitions have become dipole allowed through the doping, they can interact with fundamental KS transition. The evolution of the doped photoabsorption spectra with the interaction strength parameter is shown in Fig. 4(b). Similar to the homogeneous array, there is a strong blueshifting mode originating at $v_{6 \rightarrow 7}$. The interactions among the KS transitions lead to a gradual brightening of the additional mode at a higher energy indicated by an arrow in Fig. 4(b). The resulting excitations consisting of mixed KS transitions are examined below using the TCMs and within the Casida framework.

Transitions $\nu_{5 \rightarrow 7}$ and $\nu_{6 \rightarrow 8}$ lie below and above the line at $\omega=1.66 \mathrm{eV}$, respectively. The position reflects the energy of the photoabsorption mode $(\omega)$ relative to the difference of the eigenenergies of the initial and final states of the KS transition, i.e., $\Delta \epsilon$. It can be seen easily by comparing the noninteracting $(\lambda=0)$ and fully interacting $(\lambda=1)$ spectra in Fig. 4(b). The energy of $\nu_{5 \rightarrow 7}$ in the noninteracting spectra is less than $1.66 \mathrm{eV}$ and thus it lies below the $\omega$-probe line in the TCM. Similarly, the contribution of $v_{6 \rightarrow 8}$ to the excitation at $1.66 \mathrm{eV}$ lies above the $\omega$-probe line.

The photoabsorption bands at 1.46 and $1.66 \mathrm{eV}$ are collective excitations of multiorder KS transitions. The secondorder transitions are not single-particle excitations despite the proximity of their contributions to the $\omega$-probe line. The collectivity of the excitations is validated by inspecting the interaction among the KS transition contributions identified by the TCM.

The interaction strength or "coupling constant" between each electron-hole contribution pair is given by the offdiagonal matrix elements in $K_{i a, j b}$ [see Eq. (2)]. All KS transitions of the subset have $A_{1}$ symmetry and interact, and yet the interaction is stronger among the KS transitions with

density of the low-energy excitation are shown adjacent to the noninteracting and fully interacting spectra, respectively. (c) Wave functions of the $(5,0)$ and $(7,0)$ states along the chain axis adjacent to the array in homogeneous and $\mathrm{Cu}$-doped $10 \times 2 \mathrm{Au}$ array (left) and the respective partial transition density in the $\mathrm{Cu}$-doped array (right). 

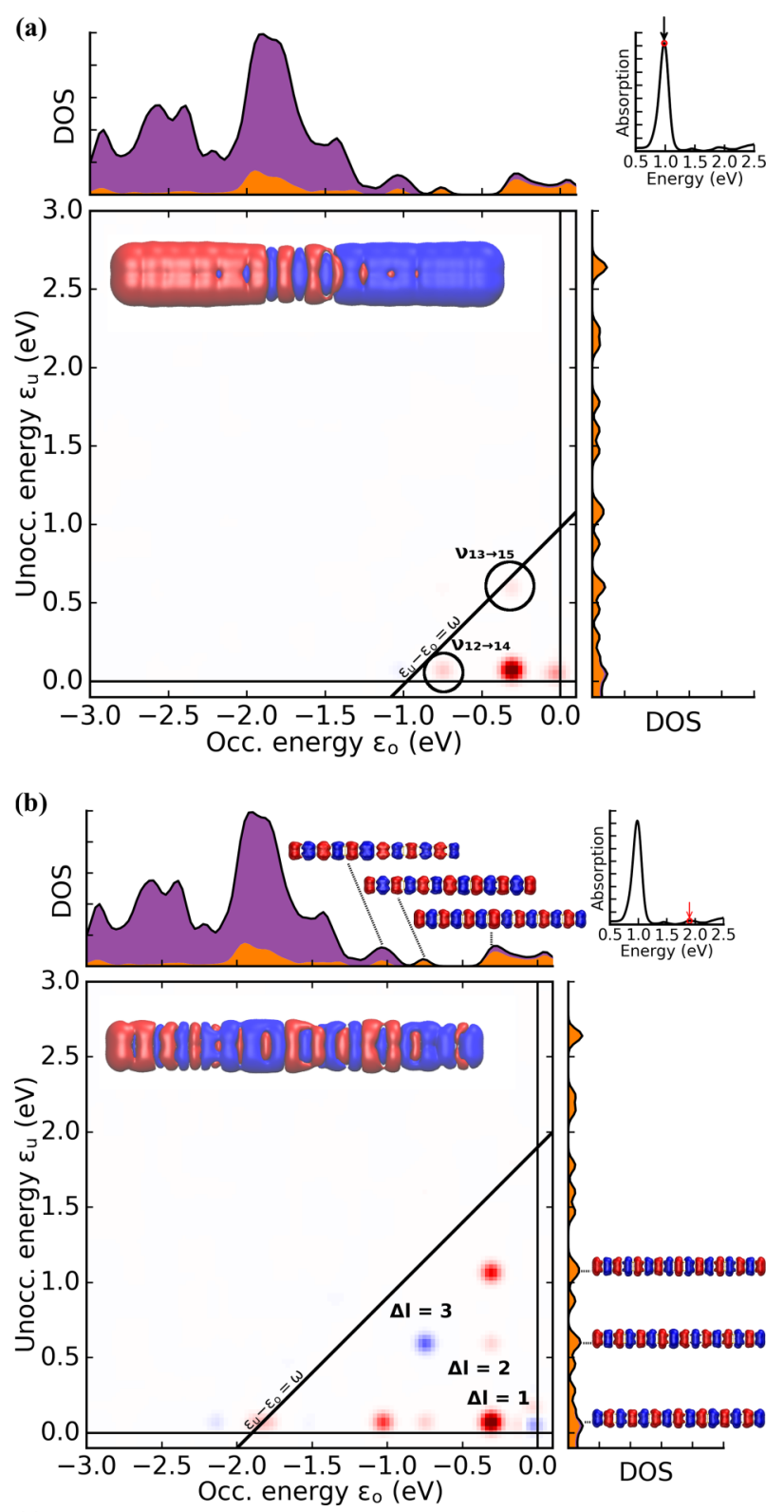

(c)

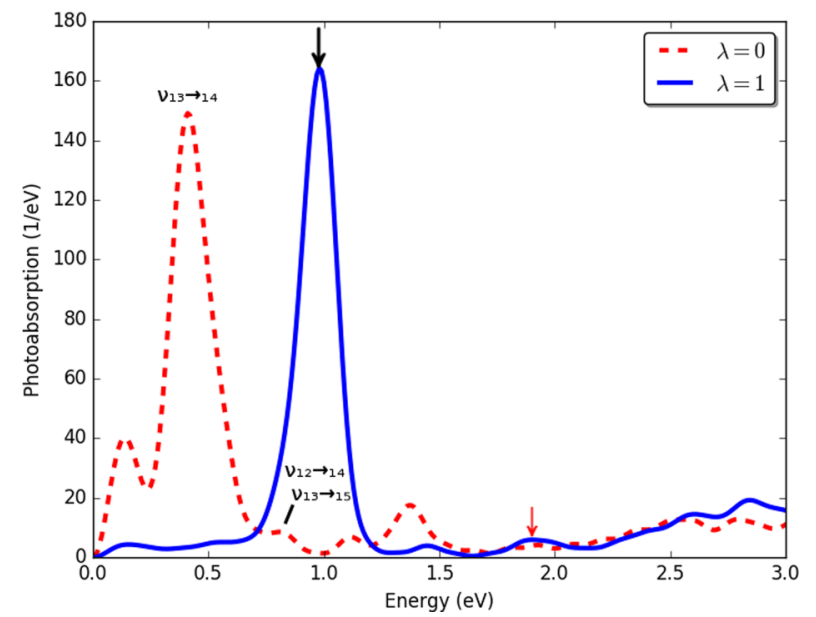

FIG. 5. Off-center $\mathrm{Cu}$-doped $20 \times 2 \mathrm{Au}$ array. There is one impurity atom in each chain and the impurities are nearest neighbors. TCM of the photoabsorption excitations at $\omega=$ (a) 0.98 and (b) $1.90 \mathrm{eV}$. The total induced density of each excitation is shown in the same order, i.e., between the two first-order transitions (having either zero or one transverse node) and between the two second-order transitions. This pairing is demonstrated in the $\lambda$ evolution of the pseudoexcitation energies of smaller subsets of KS transitions given in Fig. S5 [58].

The weak interaction between $v_{6 \rightarrow 7}$ and $v_{5 \rightarrow 7}$ does not produce two excitations around $1.5 \mathrm{eV}$ on its own. It is necessary for this to be accompanied by the strong pairing between the second-order transitions. The multiorder interactions among the electron-hole contributions are contained within the subset consisting of all the KS transitions identified in the TCM $\left(v_{5 \rightarrow 7}, v_{6 \rightarrow 8}, v_{6 \rightarrow 7}\right.$ and the HOMO $\rightarrow$ LUMO). The $\lambda$ evolution of each pseudoexcitation energy is drawn as a red dot superimposed onto the full set of possible interacting transitions in Fig. 4(b). The subset of these four KS transitions reconstitutes the energies of the excitations with full interaction at $0.41,1.46$, and $1.66 \mathrm{eV}$ [Fig. 4(b), right panel] better than the restricted subsets in Fig. S5 [58]. The excitations are the result of multiorder KS transitions induced by the $\mathrm{Cu}$ dopant. As previously mentioned in the discussion of the homogeneous arrays, the pseudoexcitations exclude the interaction with the background transitions leading to differences with the spectra with fully interaction and a strong $\lambda$ dependence of the pseudoexcitation extending from $v_{6 \rightarrow 8}$.

We now turn to the longer $20 \times 2$ array containing offcenter $\mathrm{Cu}$ dopants. The second-order transitions $\Delta l=2$ are dipole active like those in the shorter $\mathrm{Cu}$-doped array. This array will demonstrate the effect of reducing the energy difference between the interacting first- and second-order KS transitions. The energy spacing between the orders of the longer array follows from the discussion of homogeneous arrays and is validated by inspection. The KS orbitals near the Fermi level are provided in Fig. S6 [58]. Due to the narrow energy gap in the longer array, the symmetric and antisymmetric excitations coalesce into an intense mode with asymmetric line shape at $0.98 \mathrm{eV}$.

Again, contributions from second-order transitions are visible in the TCM of the molecular plasmon in Fig. 5(a). We validate that these are indeed contributions from second-order KS transitions by showing the respective KS orbitals adjacent to the TCM in Fig. 5(b). The weak mode, which is indicated by a black line in the spectrum with $\lambda=0$ in Fig. 5(c), corresponds to $v_{12 \rightarrow 14}$ and $v_{13 \rightarrow 15}(0.82$ and $0.90 \mathrm{eV}$, respectively).

The excitation is formed by the collective interaction of transitions with different orders. The added contribution from the second-order transitions does not significantly change the well-separated total induced density from the homogeneous array as seen in the TCM insets. A more drastic effect is observed in the asymmetric line shape of the mode. The photoabsorption on the low-energy shoulder is formed by the plasmon splitting between the first- and second-order transitions. See the TCM at $0.83 \mathrm{eV}$ in Fig. S4B [58] in which the second-order contributions to the photoabsorption intensity are negative (blue spots).

the inset and relevant KS orbitals are provided adjacent to the density of states. (c) Noninteracting (dashed line) and fully interacting (solid line) photoabsorption spectra. 

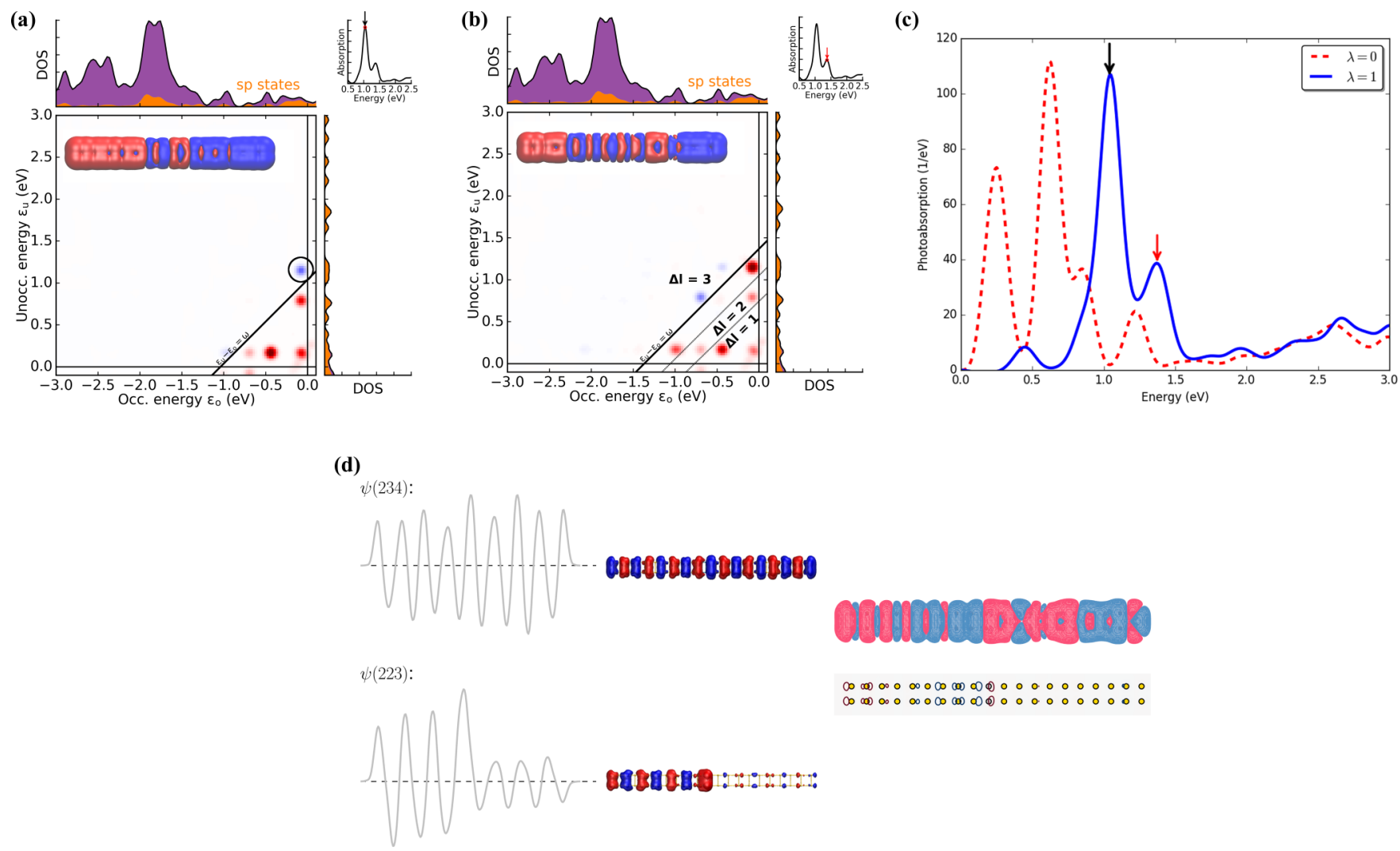

FIG. 6. Off-center Pd-doped $20 \times 2$ Au array. There is one impurity atom in each chain and the impurities are nearest neighbors. TCM of the photoabsorption excitations at (a) 1.04 and (b) $1.37 \mathrm{eV}$. The contribution from the circled transition $[\psi(223) \rightarrow \psi(234)]$ changes sign between these two excitations. The total induced density of each excitation is shown in the inset. (c) Noninteracting (dashed line) and fully interacting (solid line) photoabsorption spectra. (d) Wave functions of $\psi(223)$ and $\psi(234)$ along the chain axis adjacent to the array (left) and as isosurfaces of the KS orbitals (center) and the respective partial transition density (right).

The loss of symmetry is also displayed when the lowenergy excitations mix with the interband single-particle excitations at higher energies. A 1-2-3 pattern from the first-, second-, and third-order transition contributions is revealed in the TCM of a less intense mixed mode at $1.90 \mathrm{eV}$ in Fig. 5(b). The pattern expresses the number of possible transitions within each order, and the sum of all transitions follows the triangular number sequence in Pascal's triangle [59]. The dipole-forbidden second-order transitions are absent in the homogeneous $20 \times 2 \mathrm{Au}$ array as demonstrated in the TCM at $2.08 \mathrm{eV}$ [Fig. 3(b)]. The mixed mode at $2.08 \mathrm{eV}$ contains more contributions from single-particle excitations than the weak mixed mode at $1.90 \mathrm{eV}$ [Fig. 5(b)], which lies at the edge of the interband single-particle excitations.

Centrosymmetric $\mathrm{Cu}$-doped arrays present a unique situation in which the array is doped but has a transverse mirror plane and belongs to the $D_{2 \mathrm{~h}}$ point group. We demonstrate this situation in a 19-atom long double-chain array. As expected, the second-order transitions are dipole inactive and are absent in the TCM of the molecular plasmon at $1.05 \mathrm{eV}$ as seen in Fig. S7A [58]. For comparison, the molecular plasmon within a homogeneous array at $1.06 \mathrm{eV}$ is shown in Fig. S7B [58].

The situation of dopants placed within the arrays contrasts to the arrays formed with mixed homonuclear chains which are considered in detail elsewhere [16]. Both doped arrays have $C_{2 \mathrm{v}}$ symmetry, but in mixed arrays, the chemical heterogeneity is not along the axis of the external electric field causing the transition dipole moment of the higher-order modes along this axis to be negligible. The mixed arrays are similar to the situation when the $\mathrm{Cu}$ dopants are placed at the end of the array. Then, the even-order KS transitions $(5,0) \rightarrow$ $(7,0)$ and $(6,0) \rightarrow(8,0)$ are allowed, but their oscillator strengths are very weak as seen by the absence of even-order modes in the noninteracting spectrum in Fig. S8 [58].

\section{Pd-doped double-chain array}

The type of chemical heterogeneity affects the electronic structure of the molecular array. Doping with Pd introduces $d$ states near the Fermi level which hybridize with the $s p$ delocalized states. Weak doping is sufficient to construct new photoabsorption modes as seen in Fig. 1. In this section, we show that the formation of the modes is initiated by the $s d$ hybridization and coupling to higher-order transitions.

The long $(20 \times 2)$ Au array doped off center with $\mathrm{Pd}$ [Fig. 1(b)] has an intense photoabsorption mode at $1.04 \mathrm{eV}$ with a low-energy shoulder and a weaker but very clearly resolved mode at $1.37 \mathrm{eV}$. As described above, the higher-order modes are closer in energy to the fundamental transition in an 
array of this length. Select KS orbitals are given in Fig. S9B [58], and for convenience the order has been identified within the TCM in Fig. 6(b). It is clear the TCMs do not exhibit the same 1-2-3 pattern of the number of transition contributions within the first, second, and third orders as in the case of the $\mathrm{Cu}$-doped array in Fig. 5(b). The $s(p) d$ hybridization between the Pd $d$ states and the Au $s p$ states increases the number of possible KS transitions within the each order and increases the collectivity.

Notably, the TCMs indicate that there are contributions from the even-order transitions. Consistent with the discussion above, the point group of the array doped off center with $\mathrm{Pd}$ changes to $C_{2 \mathrm{v}}$. A weak mode from the KS transitions with $\Delta l=2$ appears at about $0.8 \mathrm{eV}$ in the noninteracting $(\lambda=0)$ photoabsorption spectra in Fig. 6(c). This energy corresponds to the low-energy shoulder in the spectrum with full interaction $(\lambda=1)$ indicating that the active even-order transition contributions split the plasmon. This splitting is confirmed by a change in the sign of the second-order transition contributions at $0.80 \mathrm{eV}$ (see TCM in Fig. S9A [58]). Such even-order transitions and low-energy shoulder are not observed in a centrosymmetric Pd-doped array (Fig. S10 [58]).

The moderately intense mode at higher energy $(1.37 \mathrm{eV})$ consists of contributions from the third-order transitions [Fig. 6(b)]. Participation from the third-order transitions was not observed in the main plasmon of homogeneous or $\mathrm{Cu}$ doped arrays [Figs. 3(a) and 5(a), respectively]. Next, we analyze one transition contribution to verify that the transitions are interacting to enhance the higher-energy excitation.

The excitation at $1.37 \mathrm{eV}$ lies just above the second- and among the third-order transition contributions. Some of the third-order transition contributions change sign between the modes at 1.04 and $1.37 \mathrm{eV}$ rather than existing as a fleeting single-particle transition on the $\omega$-probe line. The change in sign for the above transitions occurs while the low-energy transitions remain unchanged. One such transition contribution is circled in Fig. 6(a). This behavior is present also in Cu-doped arrays [compare Figs. S4A [58] and 4(a)] and has previously been described as strong coupling between a transition contribution [such as the circled one in Fig. 6(a)] and the plasmon [10]. It results in fragmentation in the spectrum $[60,61]$. Here, we demonstrate the ability of $s d$ hybridization to access the higher-order odd transitions in two-dimensional nanosystems.

Within the array, $s d$ hybridization can localize the density of a given orbital predominantly on either side of the array. KS transitions occur between these states and they participate in the collective excitations. The wave functions of the indicated transition contribution, $\psi(223)$ and $\psi(234)$, are plotted $3 \AA$ off the array edge and in the plane of the array in Fig. 6(d) (left). The notation is changed from the homogeneous and $\mathrm{Cu}$-doped array because of the complications due to the $s d$ hybridization. Along the array edge $\psi(223)$ and $\psi$ (234) have 13 and 16 longitudinal nodes, respectively. The nodes are also observed in the corresponding isosurface plots in Fig. 6(d) (center). It is clear that the amplitude of $\psi(223)$ is larger on the left end of the array, and consequently the partial induced density will also be stronger on the left end [Fig. 6(d) (right)]. The transition $\psi(223) \rightarrow \psi(234)$ is just one contribution within the collective excitation and does not localize the total transition density of the plasmon completely. The contribution from the third-order transitions is reflected in the increase of the internal nodes in the total induced density. The ends of the array continue to have the opposite charge.

The effect of the Pd remains strong even when the dopant is placed at the end of a small array. The $d$ character near the Fermi level can induce a transition dipole even at the end of an array. The effect is lost in an elongated array because the dipole moments of the third-order transitions are weak. Details are provided in the Supplemental Material and Figs. S11 and S12 [58]. To check the effect of organization within the doped arrays, two Pd atoms were substituted in alternative configurations in the $20 \times 2 \mathrm{Au}$ array. The geometries and photoabsorption spectra of arrays containing either two Pd atoms situated kitty corner in the middle of the array or one $\mathrm{Pd}$ in the middle and one $\mathrm{Pd}$ at the end of the array are given in Fig. S13 [58]. The intense photoabsorption mode has an asymmetric line shape in both configurations, suggesting the plasmon is fragmented by the various combinations of transition contributions. The contributions from transitions among the $d$-and $s d$-hybridized states to the excitations are validated with TCMs (Figs. S14 and S15 [58]) and details are provided in the Supplemental Material [58].

\section{CONCLUSIONS}

We have demonstrated how the plasmon formation in atomic arrays doped with $\mathrm{Cu}$ or $\mathrm{Pd}$ is influenced by symmetry and hybridization induced coupling to higher-order transitions using TD-DFT methods. The extended length and simplified nodal structure of double-chain arrays makes identification straightforward and yet the electronic and optical properties of chemically heterogeneous atomic arrays are more tangled than a single chain doped with a transition metal [36] and arrays of mixed homonuclear chains [16].

The generation of additional dipole-allowed transitions is dependent on the chemical composition, position, and symmetry of the doped atomic array. In these finite systems, collectivity arises through the interacting KS transition contributions, which produce composite photoabsorption modes. $\mathrm{Cu}$ dopants activate even-order KS transitions which are also strongly dependent on the interaction strength parameter $\lambda$. The antisymmetric and symmetric combination with the first-order transitions produces a new photoabsorption mode. The modes coalesce in elongated arrays due to the narrowed energy difference of the antisymmetric and symmetric combinations.

The Pd $d$ states are energetically close to the Fermi level and hybridize with the delocalized $s p$ states. The second-order KS transitions between hybridized states have a nonzero transition dipole moment and persist even when the Pd dopants are located at the end of the array. In addition, the Pd dopants allow the fundamental transition to interact with the odd higherorder transitions. The third-order transition contributions participate in collective excitations and can form an additional excitation in the centrosymmetric or noncentrosymmetric arrays. The access to the third-order KS transitions increases the plasmon fragmentation. Similar coupling between transitions has been observed in small homogeneous metal nanoparticles in which a plasmon resonance emerges from the interaction 
between confined transitions within the nanoparticle [10,61]. Here, the collective effects and access to $s d$-hybridized transitions with localized character are demonstrated in long twodimensional systems with doping. Chemical heterogeneity changes the electronic structure and orbital hybridization and enables the participation of transitions with different orders. The multiorder interactions induced by the heterogeneity dictate the plasmon formation and decay processes. The aspects in doped two-dimensional nanoarrays might be applied more generally to other chemical heterogeneous materials, such as metal nanoparticles with adsorbed molecules or ligands, structural defects, or at an interface.

The optical response of the large arrays $(19 \times 2$ and 20 $\times 2$ ) were calculated with only the TP DF-DFT code. The calculated spectra are available [52]

\section{ACKNOWLEDGMENTS}

The work was supported in part by US DOE Grant No. DE-FG02-07ER46354 (N.N., V.T., and T.S.R.) and as part of the Academy of Finland Centre of Excellence program (Project No. 251748, K.C. and M.J.P., and Project No. 312298, K.C.). T.P.R. acknowledges support from the European Union's Horizon 2020 research and innovation programme under the Marie Skłodowska-Curie Grant Agreement No. 838996. M.K. acknowledges funding from Academy of Finland under Grant No. 295602. We acknowledge computational resources provided by CSC-IT Center for Science (Finland) and by the Aalto Science-IT project (Aalto University School of Science). We also acknowledge computational resources provided by the High Performance Computing facility (STOKES) at the University of Central Florida.

K.C. and N.N. contributed equally to this work.
[1] D. Bohm and D. Pines, A collective description of electron interactions: III. Coulomb interactions in a degenerate electron gas, Phys. Rev 92, 609 (1953).

[2] K. Iida, M. Noda, K. Ishimura, and K. Nobusada, Firstprinciples computational visualization of localized surface plasmon resonance in gold nanoclusters, J. Phys. Chem. A 118, 11317 (2014).

[3] M. Kuisma, A. Sakko, T. P. Rossi, A. H. Larsen, J. Enkovaara, L. Lehtovaara, and T. T. Rantala, Localized surface plasmon resonance in silver nanoparticles: Atomistic first-principles time-dependent density-functional theory calculations, Phys. Rev. B 91, 115431 (2015).

[4] P. Koval, F. Marchesin, D. Foerster, and D. Sánchez-Portal, Optical response of silver clusters and their hollow shells from linear-response TDDFT, J. Phys.: Condens. Matter 28, 214001 (2016)

[5] P. Koval, M. Barbry, and D. Snchez-Portal, PySCF-NAO: An efficient and flexible implementation of linear response timedependent density functional theory with numerical atomic orbitals, Comput. Phys. Commun. 236, 188 (2019).

[6] J. Ma, Z. Wang, and L.-W. Wang, Interplay between plasmon and single-particle excitations in a metal nanocluster, Nat. Commun. 6, 10107 (2015).

[7] P. V. Kumar, T. P. Rossi, D. Marti-Dafcik, D. Reichmuth, M. Kuisma, P. Erhart, M. J. Puska, and D. J. Norris, Plasmoninduced direct hot-carrier transfer at metal-acceptor interfaces, ACS Nano 13, 3188 (2019).

[8] J. Ma and S. Gao, Plasmon induced electron-hole separation at the Ag/TiO2 (110) interface, ACS Nano 13, 13658 (2019).

[9] S. Bernadotte, F. Evers, and C. R. Jacob, Plasmons in molecules, J. Phys. Chem. C 117, 1863 (2013).

[10] T. P. Rossi, M. Kuisma, M. J. Puska, R. M. Nieminen, and P. Erhart, Kohn-Sham decomposition in real-time time-dependent density-functional theory: An efficient tool for analyzing plasmonic excitations, J. Chem. Theory Comput. 13, 4779 (2017)

[11] S. Malola, L. Lehtovaara, J. Enkovaara, and H. Häkkinen, Birth of the localized surface plasmon resonance in monolayer-protected gold nanoclusters, ACS Nano 7, 10263 (2013).

[12] R. Zhang, L. Bursi, J. D. Cox, Y. Cui, C. M. Krauter, A. Alabastri, A. Manjavacas, A. Calzolari, S. Corni, E. Molinari, E. A. Carter, F. J. G. de Abajo, H. Zhang, and P. Nordlander, How to identify plasmons from the optical response of nanostructures, ACS Nano 11, 7321 (2017).

[13] F. J. G. de Abajo, R. Sapienza, M. Noginov, F. Benz, J. Baumberg, S. Maier, D. Graham, J. Aizpurua, T. Ebbesen, A. Pinchuk, J. Khurgin, K. Matczyszyn, J. T. Hugall, N. van Hulst, P. Dawson et al., Plasmonic and new plasmonic materials: General discussion, Faraday Discuss. 178, 123 (2015).

[14] O. Baseggio, M. De Vetta, G. Fronzoni, M. Stener, L. Sementa, A. Fortunelli, and A. Calzolari, Photoabsorption of icosahedral noble metal clusters: An efficient TDDFT approach to largescale systems, J. Phys. Chem. C 120, 12773 (2016).

[15] T. P. Rossi, K. T. Winther, K. W. Jacobsen, R. M. Nieminen, M. J. Puska, and K. S. Thygesen, Effect of edge plasmons on the optical properties of $\mathrm{MoS}_{2}$ monolayer flakes, Phys. Rev. B 96, 155407 (2017).

[16] K. M. Conley, N. Nayyar, T. P. Rossi, M. Kuisma, V. Turkowski, M. J. Puska, and T. S. Rahman, Plasmon excitations in mixed metallic nanoarrays, ACS Nano 13, 5344 (2019).

[17] T. P. Rossi, T. Shegai, P. Erhart, and T. J. Antosiewicz, Strong plasmon-molecule coupling at the nanoscale revealed by firstprinciples modeling, Nat. Commun. 10, 3336 (2019).

[18] S. Kümmel, K. Andrae, and P.-G. Reinhard, Collectivity in the optical response of small metal clusters, Appl. Phys. B: Lasers Opt. 73, 293 (2001).

[19] D. Casanova, J. M. Matxain, and J. M. Ugalde, Plasmonic resonances in the Al13-cluster: Quantification and origin of exciton collectivity, J. Phys. Chem. C 120, 12742 (2016).

[20] S. Malola, S. Kaappa, and H. Häkkinen, Role of nanocrystal symmetry in the crossover region from molecular to metallic gold nanoparticles, J. Phys. Chem. C 123, 20655 (2019).

[21] E. Townsend and G. W. Bryant, Which resonances in small metallic nanoparticles are plasmonic?, J. Opt. 16, 114022 (2014). 
[22] L. Bursi, A. Calzolari, S. Corni, and E. Molinari, Quantifying the plasmonic character of optical excitations in nanostructures, ACS Photonics 3, 520 (2016).

[23] R. L. Gieseking, M. A. Ratner, and G. C. Schatz, Semiempirical modeling of ag nanoclusters: New parameters for optical property studies enable determination of double excitation contributions to plasmonic excitation, J. Phys. Chem. A 120, 4542 (2016)

[24] R. L. Gieseking, A. P. Ashwell, M. A. Ratner, and G. C. Schatz, Analytical approaches to identify plasmon-like excited states in bare and ligand-protected metal nanoclusters, J. Phys. Chem. C 124, 3260 (2020).

[25] B.-J. Wang, Y. Xu, and S.-H. Ke, Plasmon excitations in sodium atomic planes: A time-dependent density functional theory study, J. Chem. Phys. 137, 054101 (2012).

[26] H.-F. Yin and H. Zhang, Collectivity of plasmon excitations in small sodium clusters with planar structure, Phys. B (Amsterdam) 407, 416 (2012).

[27] J. Yan, Z. Yuan, and S. Gao, End and Central Plasmon Resonances in Linear Atomic Chains, Phys. Rev. Lett. 98, 216602 (2007).

[28] Y.-H. Huang, K.-M. Lin, T. Leung, and C. T. Chan, Plasmonlike resonances in atomic chains: A time-dependent densityfunctional theory study, Phys. Rev. B 90, 075418 (2014).

[29] J. M. Fitzgerald, S. Azadi, and V. Giannini, Quantum plasmonic nanoantennas, Phys. Rev. B 95, 235414 (2017).

[30] C. M. Krauter, S. Bernadotte, C. R. Jacob, M. Pernpointner, and A. Dreuw, Identification of plasmons in molecules with scaled $a b$ initio approaches, J. Phys. Chem. C 119, 24564 (2015).

[31] E. B. Guidez and C. M. Aikens, Origin and TDDFT benchmarking of the plasmon resonance in acenes, J. Phys. Chem. C 117, 21466 (2013).

[32] E. B. Guidez and C. M. Aikens, Quantum mechanical origin of the plasmon: From molecular systems to nanoparticles, Nanoscale 6, 11512 (2014).

[33] L. Bursi, A. Calzolari, S. Corni, and E. Molinari, Lightinduced field enhancement in nanoscale systems from firstprinciples: the case of polyacenes, ACS Photonics 1, 1049 (2014).

[34] A. Manjavacas, F. Marchesin, S. Thongrattanasiri, P. Koval, P. Nordlander, D. Sanchez-Portal, and F. J. G. de Abajo, Tunable molecular plasmons in polycyclic aromatic hydrocarbons, ACS Nano 7, 3635 (2013).

[35] N. Nilius, T. Wallis, and W. Ho, Development of onedimensional band structure in artificial gold chains, Science 297, 1853 (2002).

[36] N. Nayyar, V. Turkowski, and T. S. Rahman, Optical Generation of Collective Plasmon Modes in Small Gold Chains Induced by Doping Transition-Metal Impurities, Phys. Rev. Lett. 109, 157404 (2012).

[37] P. Hohenberg and W. Kohn, Inhomogeneous electron gas, Phys. Rev. 136, B864 (1964).

[38] W. Kohn and L. J. Sham, Self-consistent equations including exchange and correlation effects, Phys. Rev. 140, A1133 (1965).

[39] M. Kuisma, J. Ojanen, J. Enkovaara, and T. T. Rantala, KohnSham potential with discontinuity for band gap materials, Phys. Rev. B 82, 115106 (2010).
[40] J. Yan, K. W. Jacobsen, and K. S. Thygesen, First-principles study of surface plasmons on $\mathrm{Ag}(111)$ and H/Ag(111), Phys. Rev. B 84, 235430 (2011).

[41] J. Yan, K. W. Jacobsen, and K. S. Thygesen, Conventional and acoustic surface plasmons on noble metal surfaces: A timedependent density functional theory study, Phys. Rev. B 86, 241404(R) (2012).

[42] J. J. Mortensen, L. B. Hansen, and K. W. Jacobsen, Real-space grid implementation of the projector augmented wave method, Phys. Rev. B 71, 035109 (2005).

[43] J. Enkovaara, C. Rostgaard, J. J. Mortensen, J. Chen, M. Dułak, L. Ferrighi, J. Gavnholt, C. Glinsvad, V. Haikola, H. A. Hansen, H. H. Kristoffersen, M. Kuisma, A. H. Larsen, L. Lehtovaara, M. Ljungberg et al., Electronic structure calculations with GPAW: a real-space implementation of the projector augmented-wave method, J. Phys.: Condens. Matter 22, 253202 (2010).

[44] M. Walter, H. Häkkinen, L. Lehtovaara, M. Puska, J. Enkovaara, C. Rostgaard, and J. J. Mortensen, Time-dependent density-functional theory in the projector augmented-wave method, J. Chem. Phys. 128, 244101 (2008).

[45] P. E. Blöchl, Projector augmented-wave method, Phys. Rev. B 50, 17953 (1994).

[46] A. H. Larsen, J. J. Mortensen, J. Blomqvist, I. E. Castelli, R. Christensen, M. Dułak, J. Friis, M. N. Groves, B. Hammer, C. Hargus, E. D. Hermes, P. C. Jennings, P. B. Jensen, J. Kermode, J. R. Kitchin et al., The atomic simulation environment-a python library for working with atoms, J. Phys.: Condens. Matter 29, 273002 (2017).

[47] A. H. Larsen, M. Vanin, J. J. Mortensen, K. S. Thygesen, and K. W. Jacobsen, Localized atomic basis set in the projector augmented wave method, Phys. Rev. B 80, 195112 (2009).

[48] E. Runge and E. K. U. Gross, Density-Functional Theory for Time-Dependent Systems, Phys. Rev. Lett. 52, 997 (1984).

[49] M. E. Casida, Time-dependent density functional response theory for molecules, in Recent Advances in Density Functional Methods, Part I, edited by D. P. Chong (World Scientific, Singapore, 1995), p. 155.

[50] J. P. Perdew, K. Burke, and M. Ernzerhof, Generalized Gradient Approximation Made Simple, Phys. Rev. Lett. 77, 3865 (1996).

[51] K. Yabana and G. F. Bertsch, Time-dependent local-density approximation in real time, Phys. Rev. B 54, 4484 (1996).

[52] The atomic structures and the calculated photoabsorption spectra are available at are K. Conley et al., Data for "Plasmon excitations in chemically heterogeneous nanoarrays", https:// doi.org/10.5281/zenodo.3860069 (2020).

[53] M. J. Frisch, G. W. Trucks, H. B. Schlegel, G. E. Scuseria, M. A. Robb, J. R. Cheeseman, J. A. Montgomery, Jr., T. Vreven, K. N. Kudin, J. C. Burant, J. M. Millam, S. S. Iyengar, J. Tomasi, V. Barone, B. Mennucci et al., Gaussian 03, Revision D.01, Gaussian, Inc., Wallingford, CT, 2004.

[54] A. D. Becke, Density-functional thermochemistry. III. The role of exact exchange, J. Chem. Phys. 98, 5648 (1993).

[55] J. P. Perdew and W. Yue, Accurate and simple density functional for the electronic exchange energy: Generalized gradient approximation, Phys. Rev. B 33, 8800 (1986).

[56] J. P. Perdew, in Electronic Structure of Solids, edited by P. Ziesche and H. Eschrig (Academic, Berlin, 1991). 
[57] P. J. Hay and W. R. Wadt, Ab initio effective core potentials for molecular calculations. Potentials for $\mathrm{K}$ to Au including the outermost core orbitals, J. Chem. Phys. 82, 299 (1985).

[58] See Supplemental Material at http://link.aps.org/supplemental/ 10.1103/PhysRevB.101.235132 for additional information on the antisymmetric combinations, centrosymmetric arrays, and arrays doped at the end. Spectra obtained using Gaussian 03 are also provided.
[59] V. E. Hoggatt, Jr. and M. Bicknell, Triangular numbers, Fibonacci Q. 12, 221 (1974).

[60] C. Yannouleas, R. A. Broglia, M. Brack, and P. F. Bortignon, Fragmentation of the Photoabsorption Strength in Neutral and Charged Metal Microclusters, Phys. Rev. Lett. 63, 255 (1989).

[61] C. Yannouleas and R. A. Broglia, Collective and single-particle aspects in the optical response of metal microclusters, Phys. Rev. A 44, 5793 (1991). 